\title{
Can Certain Foods Cause Gastrointestinal Symptoms in Korean Patients With Irritable Bowel Syndrome?
}

\author{
Kwangwoo Nam and Jeong Eun Shin* \\ Department of Internal Medicine, Dankook University College of Medicine, Cheonan, Chungcheongnam-do, Korea
}

Article: Self-reported food intolerance in Korean patients with irritable bowel syndrome

Lee HJ, Kim HJ, Kang EH, et al

(J Neurogastroenterol Motil 2019;25:222-232)

Irritable bowel syndrome (IBS) is a common functional gastrointestinal (GI) disorder, which is characterized by abdominal pain and altered bowel habits without structural abnormalities. IBS is not a life-threatening condition, however it can impair patients' quality of life and mental health, ${ }^{1}$ resulting in considerable economic burden on the patients. ${ }^{2,3}$ Recent studies have emphasized the relationship between IBS and food intake, and many IBS patients want to know specific foods which aggravate GI symptoms, and receive proper dietary management. ${ }^{4}$ High fermentable oligo-, di-, mono-saccharides, and polyols (FODMAP) diet is regarded as an important aggravating factor of IBS. Indigestion of FODMAPs can increase the delivery of readily fermentable substrates to the distal small intestine and colon, increasing bowel motility, visceral sensitivity, immune reaction, and permeability. In previous studies, low-FODMAP diet is reported to improve GI symptoms in patients with IBS. ${ }^{5,6}$ Although there is concern about the effect of long-term low-FODMAP diet on patients' nutritional status, ${ }^{6}$ lowFODMAP diet is now recommended as an important therapeutic intervention in patients with IBS. ${ }^{7}$ However, most studies were performed in Western countries, and to date, data of Asian patients with IBS are lacking. Considering the different eating habits, lifestyle, and cooking methods between Eastern and Western countries, the investigation regarding food intolerance of Asian patients with IBS is needed, especially in Korea.

In this issue of the Journal of Neurogastroenterology and Motility, the authors performed a multicenter study to investigate the prevalence of food intolerance and the association between specific foods and GI symptoms in patients with IBS. ${ }^{8}$ In their study, 393 Korean subjects (including 101 IBS patients, 167 symptomatic non-IBS subjects, and 125 control subjects) from 9 tertiary centers were enrolled. Self-reported food intolerance questionnaire which included 119 food items (6 food groups) was used. As a result, there was high prevalence of food intolerance in patients with IBS (79.2\%) compared to control subjects (44.8\%), and most problematic foods were high-fat foods (25.0\%), gluten foods (23.8\%), spicy foods (15.0\%), and dairy products (15.0\%). Specific foods containing high-FODMAP (63.4\%) and high-fat (48.5\%) were related to GI symptoms. Compared to control subjects, patients with IBS reported a greater number of problematic foods compared to control subjects (median number, 3 vs 0 ), which is consistent with the

Received: March 13, 2019 Revised: None Accepted: March 14, 2019

() This is an Open Access article distributed under the terms of the Creative Commons Attribution Non-Commercial License (http://creativecommons. org/licenses/by-nc/4.0) which permits unrestricted non-commercial use, distribution, and reproduction in any medium, provided the original work is properly cited.

*Correspondence: Jeong Eun Shin, MD, PhD

Department of Internal Medicine, Dankook University College of Medicine, 201 Manghyang-ro, Dongnam-gu, Cheonan,

Chungcheongnam-do 31116, Korea

Tel: +82-41-550-3052, Fax: +82-41-556-3256, E-mail: dreun1028@daum.net 
Western study. ${ }^{9}$ Gas distension/bloating (36.3\%) and loose stool/ diarrhea $(28.8 \%)$ are the most commonly reported food-induced GI symptoms. Spicy foods were associated with abdominal pain, and most other foods were associated with gas problems and loose stools. IBS subtype is not associated with food-related GI symptoms, and patients with IBS tend to restrict their food to minimize their symptoms compared to control subjects (71.3\% vs $30.9 \%$, $P<0.001)$.

The meaning of this study is that it is the first identification of the intolerance to specific foods in Korean patients with IBS. The authors showed the relationship between high-FODMAP/high-fat diet and GI symptoms. Traditional Korean foods have FODMAPs although the content is lower than Western foods. In addition, eating habits tend to be westernized in Korea, and many fast foods and processed foods have high content of FODMAPs and fat. Considering the high rate of high-FODMAP diet related GI symptoms in this study, low-FODMAP diet is also important in Korean patients with IBS. The authors categorized 55 problematic and 64 rarely problematic food items which can be a good clinical reference to make specific dietary management to Korean IBS patients.

However, the questionnaire used in this study had not been validated, and the recipe of each food item was not standardized. Spicy foods may contain high-FODMAP and high-fat ingredients, and abdominal pain related to spicy foods can be individually different. Thus it is difficult to recommend avoidance of spicy foods to all patients with IBS. In the present study, approximately $42.6 \%$ of IBS patients were over 60 years of age, and these patients were usually accompanied by chronic diseases, such as diabetes, hypertension, neurodegenerative diseases, or even multiple comorbidities. In addition to these comorbidities, medications used to control these conditions may cause IBS-like symptoms, which may bias the selection of patients. Also, patients' conditions that affect food intolerance such as combined non-GI organic disease and past surgical history were not evaluated. In a previous study, overlap between functional dyspepsia and IBS which mimics food-related symptoms is reported, ${ }^{10}$ and it may affect the results of this study. Thus, a following well-controlled double blind challenge is needed to confirm the relationship between specific foods and GI symptoms, and generalize dietary management strategy to patients with IBS.

Financial support: The present research was conducted by the research fund of Dankook University in 2018.

\section{Conflicts of interest: None.}

Author contributions: Kwangwoo Nam drafted the manuscript and Jeong Eun Shin revised the manuscript.

\section{References}

1. Kopczynska M, Mokros L, Pietras T, Malecka-Panas E. Quality of life and depression in patients with irritable bowel syndrome. Prz Gastroenterol 2018;13:102-108.

2. Zhang F, Xiang W, Li CY, Li SC. Economic burden of irritable bowel syndrome in China. World J Gastroenterol 2016;22:10450-10460.

3. Canavan C, West J, Card T. Review article: the economic impact of the irritable bowel syndrome. Aliment Pharmacol Ther 2014;40:1023-1034.

4. Halpert A, Dalton CB, Palsson O, et al. What patients know about irritable bowel syndrome (IBS) and what they would like to know. National Survey on Patient Educational Needs in IBS and development and validation of the Patient Educational Needs Questionnaire (PEQ). Am J Gastroenterol 2007;102:1972-1982.

5. Magge S, Lembo A. Low-FODMAP diet for treatment of irritable bowel syndrome. Gastroenterol Hepatol (N Y) 2012;8:739-745.

6. Altobelli E, Del Negro V, Angeletti PM, Latella G. Low-FODMAP diet improves irritable bowel syndrome symptoms: a meta-analysis. Nutrients Published Online First: 26 Aug 2017. doi:10.3390/nu9090940.

7. Song KH, Jung HK, Kim HJ, et al. Clinical practice guidelines for irritable bowel syndrome in Korea, 2017 revised edition. J Neurogastroenterol Motil 2018;24:197-215.

8. Lee HJ, Kim HJ, Kang EH, et al. Self-reported food intolerance in Korean patients with irritable bowel syndrome. J Neurogastroenterol Motil 2019;25:222-232.

9. Böhn L, Störsrud S, Törnblom H, Bengtsson U, Simrén M. Selfreported food-related gastrointestinal symptoms in IBS are common and associated with more severe symptoms and reduced quality of life. Am J Gastroenterol 2013;108:634-641.

10. Fujiwara Y, Arakawa T. Overlap in patients with dyspepsia/functional dyspepsia. J Neurogastroenterol Motil 2014;20:447-457. 